\title{
Estrategia didáctica con enfoque ciencia, tecnología, sociedad y ambiente, para la enseñanza de aspectos de bioquímica
}

\author{
Leonardo Martínez*
Álvaro Pío Rojas Duarte** \\ Leonardo Martínez*
Álvaro Pío Rojas Duarte** \\ Artículo recibido: 12-12-2005 y aprobado:26-04-2006 \\ Strategy based on science approach, \\ technology, society and environment to the \\ teaching of biochemistry topics
}

Resumen: En este artículo se presentan los resultados obtenidos a partir de la implementación de una estrategia didáctica con base en el enfoque Ciencia, Tecnología, Sociedad y Ambiente (CTSA), para contribuir con el aprendizaje de conceptos de bioquímica y con la formación de futuros profesores críticos, responsables y capaces de tomar decisiones frente a los problemas sociales y ambientales que enfrenta el país, como es el caso de las fumigaciones con glifosato. Los estudios CTSA en la enseñanza de las ciencias han permitido la construcción de currículos innovadores, el desarrollo de materiales didácticos y el mejoramiento de las prácticas docentes. Estos estudios hacen posible superar la imagen neutral de la ciencia, al establecer mayores relaciones entre la ciencia y la tecnología, con la cultura, la política, la sociología y la ética, y así abordar los problemas de la educación en ciencias.

Palabras clave: Estrategia didáctica, CTSA, bioquímica, casos simulados.

Summary: This article shows the results obtained from the implementation of a strategy based on science approach, technology, society and environment (STSE). This study contributes to the learning of concepts about biochemistry and to the education of critical and responsible teachers, able to make decisions when facing social and environmental problems in the country. The STSE studies in science teaching have been valuable in the construction of innovative curricula, in materials design and in teaching practice.These studies made possible the improvement in science image, and established logical relationship among science, technology culture and politics in order to solve educational problems in science teaching.

Key words: Strategy, STSE, biochemistry, feigned cases.

\footnotetext{
Profesor, Departamento de Química, Universidad Pedagógica Nacional.lemartinez@uni.pedagogica.edu.co.

** Profesor IED Miguel Antonio Caro, Bogotá. arojasdu@redp.edu.co.
}

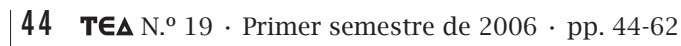




\section{Introducción}

La investigación en el campo de la didáctica de las ciencias ha identificado diversidad de inconvenientes en los procesos de enseñanza aprendizaje; por ejemplo, la estructura lógica de contenidos conceptuales, el nivel de exigencia formal de los mismos, así como la importancia de los conocimientos previos y de las preconcepciones de los estudiantes (Campanario, 1999).

Si bien es cierto que se han alcanzado avances significativos en el tema de las relaciones enseñanza y aprendizaje, en la caracterización de las concepciones alternativas de los estudiantes de los diferentes niveles educativos frente a los distintos conceptos de prácticamente la mayoría de disciplinas científicas (física, biología, química y geología), en opinión de Carrascosa (2005) y Bucat (2005) el problema actual es qué hacer con dichas concepciones y qué papel deben desempeñar en el aprendizaje de las ciencias, por lo cual la preocupación estaría en la construcción de diversas estrategias de enseñanza y aprendizaje (AP) que interesen e involucren activamente al educando en la apropiación de la ciencia y la tecnología. Así, la elaboración de estrategias de enseñanza puede poseer diferentes enfoques; entre otros están: el histórico (Libres, Comesaña y Tojo, 2001); el cognitivo (Galavoskky y otros, 2003; Jhonstone, 1997; Moreira, Greca y Rodríguez, 2004; Gutiérrez, 2004) y el enfoque Ciencia, Tecnología, Sociedad y Ambiente (CTSA) (Acevedo, 1996a; Gordillo y Acevedo, 2005; Garritz, 1994; Manassero, y Acevedo, 2001; Membiela, 1995, 1997; Solbes y Vilches, 1992).
El enfoque CTSA se perfila como una importante promesa para la enseñanza y el aprendizaje de las ciencias, en la medida en que puede favorecer la integración de las dimensiones actitudinales, axiológicas y cognitivas de los estudiantes, al igual que propender por un trabajo interdisciplinario. Por el momento, son varias las propuestas que han demostrado que el enfoque favorece un cambio actitudinal (Catebiel, 2003; Solbes y Vilches, 1998); también se han propuesto estrategias didácticas para favorecer el aprendizaje de conceptos científicos desde la perspectiva interdisciplinar CTSA (Guerrero y Cabrera, 2004).

Otro elemento que apoya el enfoque CTSA proviene de la educación ambiental (EA), cuyas referencias históricas (Estocolmo, 1972; Tblisi, 1977; Moscú, 1987; Río de Janeiro, 1992; Foro Global, 1992; Salónica, 1997) afirman que, para lograr los objetivos más relevantes de la misma, es necesario facilitar un papel activo de la persona que aprende en lo referente a su propio proceso de aprendizaje y, como consecuencia de ello, plantea la necesidad de que el papel del profesorado se caracterice fundamentalmente por facilitar las condiciones necesarias para ello (Novo, 1998, citado por Guruceaga y González, 2004). Además, los grandes problemas ambientales han develado la emergencia planetaria, y con ella la necesidad de dirigir mayores esfuerzos a la educación científica y tecnológica de los ciudadanos (Edwards, et al., 2004). Así pues, es el espacio adecuado para seguir profundizando y adecuando aspectos del enfoque CTSA con el fin de desarrollar actividades y 
propuestas que permitan al estudiante mejorar la imagen empobrecida de la ciencia (Solbes y Vilches, 2004), favorecer nuevos aprendizajes y contribuir a su formación ciudadana crítica, acorde con las necesidades del mundo actual.

El planteamiento de estrategias innovadoras desde el enfoque CTSA puede dinamizar el proceso de enseñanzaaprendizaje de las ciencias, como ya ha sido expuesto por Membiela, (1995, 1997) indicando ejemplos de implementación de proyectos CTSA, como el Apqua, Chemcom, Proyecte Gaia y Aces, que han tenido lugar en diferentes niveles educativos. Así mismo, el enfoque ha desempeñado un papel importante en el diseño de propuestas curriculares para la educación en ciencias, que a su vez promueve el diseño de estrategias de enseñanza, mediante las cuales el aprendizaje sea dinámico y rompa la monotonía de los modelos tradicionales.

Gran parte de los esfuerzos hechos en la elaboración de currículos CTS, (Ciencia, Tecnología y Sociedad) han estado centrados en la educación básica y media; sin embargo, hay unos pocos trabajos en el nivel universitario que han incorporado este enfoque, como es el caso del proyecto Química del consumidor desarrollado en la Universidad de Miami y el proyecto Cepup (Chemical Education for public Understanding Program) de la Universidad de California, ambos proyectos citados por Garritz (1994) como contribuciones importantes para la educación y enseñanza-aprendizaje de la química.

Aunque en Colombia son pocas las experiencias de enseñanza y aprendizaje en el nivel universitario que han incorporado el enfoque CTS, se destaca el trabajo de Valderrama y Jiménez (2004), quienes diseñan e implementan un curso de Tecnología y Sociedad, para 300 estudiantes de todas las facultades de la Universidad de los Andes, alcanzando en un año de experiencia el desarrollo de controversias simuladas frente a una eventual escasez de agua en Bogotá y sobre el futuro de las tecnologías de la información. La implementación del curso transcendió a la conformación de grupos interdisciplinarios de investigación de la Facultad de Ingeniería de la Universidad de los Andes en busca de respuestas a la actual necesidad de una reflexión educativa sobre el papel y la importancia de la tecnología en la realidad nacional.

En dirección a aportar en los procesos de innovación en la enseñanza-aprendizaje de las ciencias, y en particular de la bioquímica en el nivel universitario, en consonancia con el enfoque CTSA, que permite superar la desarticulación existente entre los contenidos que se enseñan en química y los aspectos de la cotidianidad que viven los estudiantes, se plantea la siguiente pregunta que orienta la investigación: ¿Qué características debe presentar una estrategia didáctica que contribuya al aprendizaje de conceptos de bioquímica y a la formación de futuros profesores críticos, responsables y capaces de tomar decisiones frente a problemas sociales y ambientales que enfrenta el país, como es el caso de las fumigaciones con glifosato?

\section{Marco teórico}

Hasta finales de la segunda guerra mundial no era usual la reflexión a propósito del papel que representaban 
la ciencia y la tecnología en la sociedad; no obstante, las complejas causas y consecuencias que arrojó esta guerra, y en especial lo que deparó posteriormente la guerra fría, llamó la atención a los gobiernos occidentales sobre la importancia social que tenían la ciencia y la tecnología, convirtiéndose en piedra angular de la seguridad nacional de los estados y de su consecuente desarrollo económico. El acelerado desarrollo de la ciencia y la tecnología en estos países trajo consigo consecuencias nefastas para el ambiente y la salud de los seres humanos; por ejemplo, se incrementó la contaminación del aire y de la atmósfera, y con ello se aceleró el efecto invernadero, se aumento el vertimiento de sustancias tóxicas de las industrias a los recursos hídricos, se intensificaron los programas de armamento químico y biológico, situaciones que pusieron en riesgo la vida de muchas personas. En respuesta a todo esto emergió el movimiento Ciencia, tecnología-sociedad, por parte de grupos ambientales y sociales que reclamaban acciones políticas para el uso responsable de la ciencia y la tecnología. Las demandas de este movimiento desarrolladas especialmente en Norteamérica constituyen una primera vertiente del origen de CTS (Science, Technology and Society), según Manassero y Acevedo (2001), más activista, política y pragmática (que pone el énfasis en los impactos sociales y ambientales de los productos científicos y tecnológicos). Otra vertiente, según los mismos autores, correspondería a la perspectiva europea caracterizada por los estudios sociales de la ciencia con una fuerte inclinación académica, que según González, López y Luján (1997) tendrían origen en el programa fuerte de la sociología del conocimiento científico (SCC) que proponen y desarrollan autores como Barry Barnes y David Bloor de la Universidad de Edimburgo durante la década de los años setenta. Los trabajos de estos autores vendría a ser una lectura crítica de filósofos de las ciencias como Kuhn, pasando a entender la ciencia como un producto de sus circunstancias históricas y culturales, como un resultado de la lucha de intereses entre clases y grupos sociales heterogéneos.

Los estudios CTS en sus dos dimensiones incursionarían en la educación (educación CTS) como un movimiento preocupado por responder a la falta de alfabetización científica y tecnológica de los ciudadanos, por medio de propuestas educativas innovadoras que tienen como finalidad construir conocimientos y valores que favorezcan la participación ciudadana en la evaluación y el control de las implicaciones sociales y ambientales.

La educación CTS comienza a constituirse como una alternativa a la tendencia de la enseñanza de las ciencias de mediados del siglo XX, centrada en los contenidos con un fuerte enfoque reduccionista, técnico y universal. Las prácticas de los docentes de ciencias recaían, la mayoría de las veces, en un conjunto de elementos que refuerzan el aprendizaje memorístico lleno de datos descontextualizados; de allí que la educación CTS permita mejorar ciertos aspectos de la enseñanza y del aprendizaje de las ciencias, teniendo en cuenta diversas variables que contribuyen a motivar a los estudiantes para la reconstrucción de su conocimiento. Las unidades curriculares que han sido 
pensadas desde un enfoque CTSA tienen varios propósitos, por ejemplo, generar actitudes de formación personal en relación con el ambiente y la calidad de vida, permitirle al estudiante tomar decisiones con respecto a las temáticas trabajadas, teniendo en cuenta aspectos científicos, técnicos, económicos y políticos, que posibilita la participación individual y social de los estudiantes de una manera responsable y autónoma.

Así, el enfoque CTSA surge como una alternativa diferente y apropiada, mediante la cual el proceso de enseñanza-aprendizaje de las ciencias ya no se concibe como un proceso memorístico, sino más bien como una estrategia motivadora y enriquecedora para el estudiante.

Precisamente la estrategia de enseñanza y aprendizaje CTS que se propone en este artículo pretende contribuir al aprendizaje de conceptos de bioquímica y a la formación de futuros profesores críticos, responsables y capaces de tomar decisiones frente a los problemas sociales y ambientales que enfrenta el país, como es el caso de las fumigaciones con glifosato. Para este fin se diseñan y se desarrollan las actividades de acuerdo con la metodología de casos simulados (Gordillo y Osorio, 2003). Esta simulación educativa hace parte de una de las metodologías más atractivas para el aprendizaje del estudiante, porque permite desarrollarlo a través de debates, habilidades argumentativas, participativas y propositivas, rompiendo con un ambiente rutinario manejado en el aula de clase. De igual forma, promueve la confrontación de ideas, informaciones y argumentaciones, generando controversia acerca de muchos problemas sociales, ambientales y tecnológicos, dentro de los cuales están presentes algunos conceptos científicos. Por tanto, los casos simulados consisten en una articulación educativa de controversia pública con el desarrollo tecno-científico, con implicaciones sociales y medioambientales, en la que se trata de utilizar una noticia ficticia pero verosímil que permita plantear controversia en los estudiantes, para que ellos investiguen cómo varios actores sociales influyen en la noticia y las complementen con sus ideas.

Para plantear un caso simulado en el aula de clase se requiere:

- Compartir la noticia a la cual se le da controversia y presentar con ella los diversos actores sociales.

- Discutir el tema de estudio para analizar las ideas y los conocimientos iniciales de los estudiantes.

- Repartir los actores sociales para que estudien y busquen la razón del comportamiento de cada actor frente al tema. Al mismo tiempo se selecciona un grupo conciliador que estará encargado de sintetizar los acuerdos en el debate establecido.

- Fuera de la controversia y el debate, se deja un tiempo para el diálogo sobre el juicio emitido por el grupo conciliador.

En los casos simulados, el profesor se encarga de buscar la noticia de la cual quiere generar controversia y reparte los actores sociales; por su parte, el estudiante debe documentarse desde el actor social al cual representa, ampliando sus argumentos con el fin de producir conocimientos que persuadan a los demás actores sociales. Esta metodología permite traer la realidad al salón de clase haciendo posible la reflexión de 
problemas sociales relevantes, con miras a aportar posibles soluciones sobre los mismos.

\section{Desarrollo del tema}

La estrategia didáctica desde el enfoque CTSA se implementó con once estudiantes de Licenciatura en Química, de la Universidad Pedagógica Nacional, que cursaban noveno y décimo semestre de la carrera, y que a su vez participaban del espacio académico de Bioquímica II.

La estrategia se desarrolló en dos etapas. En la primera, se aplicó un instrumento (véase anexo 1) que contenía 18 ítems, elaborados de acuerdo con una prueba de tipo Likert. De los 18 ítems, seis buscaban auscultar la disposición positiva o negativa de los estudiantes para establecer relaciones entre CTSA. Seis ítems fueron diseñados para caracterizar concepciones alternativas de los estudiantes frente a conceptos de bioquímica asociados al tema del metabolismo vegetal en consonancia con la problemática ambiental de las fumigaciones y seis ítems orientados a determinar el grado de conocimiento de los estudiantes hacia la problemática ambiental que implica las fumigaciones con glifosato.

Además de lo anterior, en la primera etapa se llevó a cabo una reflexión sobre las fumigaciones aéreas con glifosato en Colombia, cuya intención era propiciar el surgimiento de la situación problema que suscita el desarrollo de una controversia, para lo cual se presentaron tres pequeñas noticias (véase anexo 2) que daban a conocer denuncias y dificultades surgidas a partir de las fumigaciones con glifosato, las noticias son reales y se ubican en dos tiempos distintos.
Estudiadas las noticias reales, se da paso al desarrollo de la controversia con relevancia social en la cual participan diferentes actores sociales que son representados por grupos de estudiantes. Esto constituye el caso simulado, que se desenvuelve en la segunda etapa de la estrategia, para lo cual se tuvo como referente teórico la propuesta de Gordillo y Osorio (2003), quienes sugieren la implementación de casos simulados CTS, consistentes en una articulación educativa de controversias públicas relacionadas con desarrollos tecnocientíficos con implicaciones sociales o medioambientales. A diferencia de estos autores, las noticias que se trabajan no son ficticias sino que hacen parte de la realidad colombiana.

\section{Primera etapa}

En el instrumento presentado en el anexo 1, se puede apreciar que los ítems $1,2,4$, son afirmaciones positivas o adecuadas para abordar las relaciones CTSA, mientras que los ítems 3, 5 y 6 son afirmaciones negativas o poco adecuadas para abordar estas relaciones. Por su parte, los ítems 7, 8 y 10 son apreciaciones conceptuales adecuadas de bioquímica vegetal, mientras que los ítems 9, 11 y 12 son apreciaciones conceptuales inadecuadas ${ }^{1}$ Por último,

\footnotetext{
1 Si bien es cierto la escala tipo Likert es frecuentemente usada para detectar actitudes, también ha sido utilizada para establecer la tendencia que tiene un grupo de personas hacia afirmaciones adecuadas o inadecuadas de contenidos científicos como los que implica el metabolismo vegetal.Por ejemplo Gallego y Pérez (1999) elaboro un instrumento tipo Likert que contiene afirmaciones acerca de contenidos generales de química como la acidez y la síntesis química.
} 
los ítems 13, 14 y 16 son frases que demuestran conocimiento de la problemática ambiental causada por las fumigaciones, y los ítems 15, 17 y 18 son frases que demuestran desconocimiento de la problemática ambiental causada por las fumigaciones.

Para efectos de facilitar el análisis de los resultados, se decidió establecer los rangos que se muestran en la tabla 1, en donde la máxima puntuación que podría obtener cada estudiante, tanto para las afirmaciones positivas como negativas, de las tres categorías, sería de 15. Además, hay que tener en cuenta, de acuerdo con las opciones dadas en el instrumento, que las puntuaciones altas son favorables para el caso de las afirmaciones positivas y negativas, por lo cual se realizó la conversión de los puntajes totales de las afirmaciones negativas, tal como es señalado por Del Rincón, Arnal, Latorre y Sans (1995), aplicando la siguiente ecuación:

(grados de la escala +1$)-$ puntuación obtenida = puntaje convertido para las afirmaciones negativas,

Como los grados de la escala son 5 , para el caso de un puntuación de 5 la conversión daría 1 , lo cual deja claro que puntuaciones totales altas (11-15) en los ítems negativos indicarán que los estudiantes no están de acuerdo con la expresión, mientras que puntaciones totales bajas (0-15) indicarán que los estudiante están de acuerdo con ella.

\begin{tabular}{lcc}
\hline \multicolumn{1}{c}{ Categorías de análisis } & No. Categoría & Rango \\
\cline { 1 - 2 } Disposición negativa para establecer relaciones entre CTSA & 1 & $0-5$ \\
\cline { 1 - 2 } Apreciaciones conceptuales erróneas de bioquímica vegetal & 2 \\
\hline $\begin{array}{l}\text { Desconocimiento de la problemática ambiental causada por las } \\
\text { fumigaciones. }\end{array}$ & 1 \\
\hline $\begin{array}{l}\text { Se les dificulta establecer algunas relaciones entre CTSA } \\
\text { Se les dificulta establecer apreciaciones conceptuales correctas de } \\
\text { bioquímica vegeta }\end{array}$ & 2 \\
\hline $\begin{array}{l}\text { Se les dificulta establecer la problemática ambiental causada por las } \\
\text { fumigaciones }\end{array}$ & 3 \\
\hline $\begin{array}{l}\text { Disposición positiva para establecer relaciones entre CTSA } \\
\text { gaciones. }\end{array}$ & 1 \\
\hline \begin{tabular}{l} 
Apreciaciones conceptuales correctas de bioquímica vegetal \\
\hline
\end{tabular} & 2 \\
\hline
\end{tabular}

Tabla 1. Categorías y rangos de análisis. 
En la figura 1 se observan las puntuaciones totales de las afirmaciones negativas y positivas de acuerdo con cada estudiante para la categoría de análisis 1 y según la tabla 1. En la figura se aprecia, que aunque la mayoría (10) de los estudiantes presentan una dispo- sición positiva para establecer relaciones entre CTSA, todos los estudiantes frente a las afirmaciones negativas se les dificulta establecer algunas relaciones entre CTSA, ya que no descartan con propiedad dichas apreciaciones ubicándose en el rango de 6 a 10 puntos.

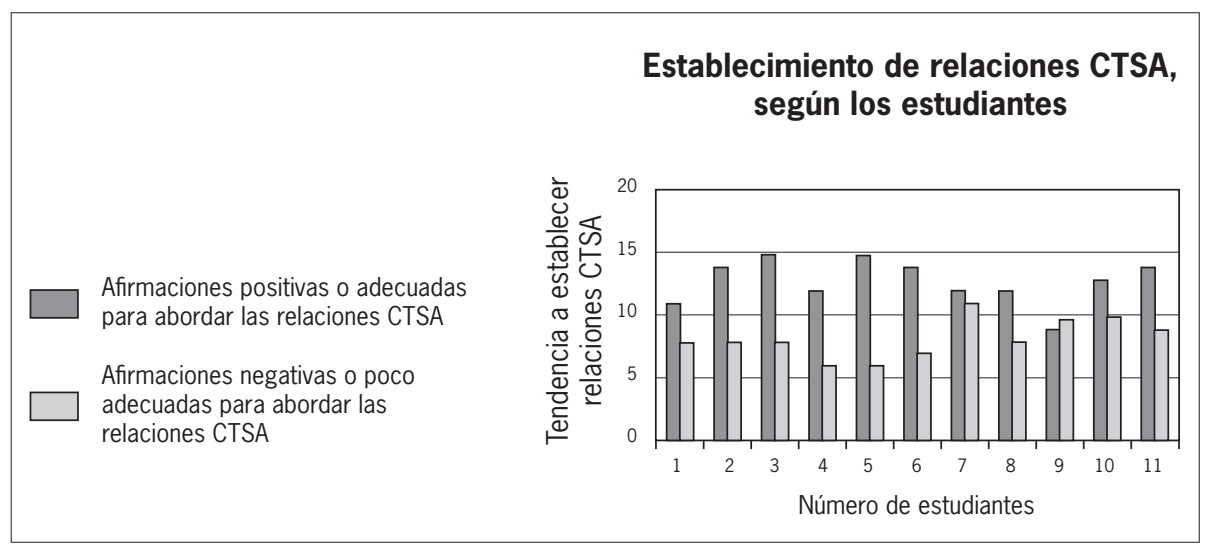

Figura 1.

Por su parte, en la figura 2 se indican las puntuaciones totales de las afirmaciones negativas y positivas de acuerdo con cada estudiante para la categoría de análisis 2 y según la tabla 1 . En figura se aprecia una tendencia de los estudiantes a establecer aspectos inadecuados de bioquímica vegetal (6-10).

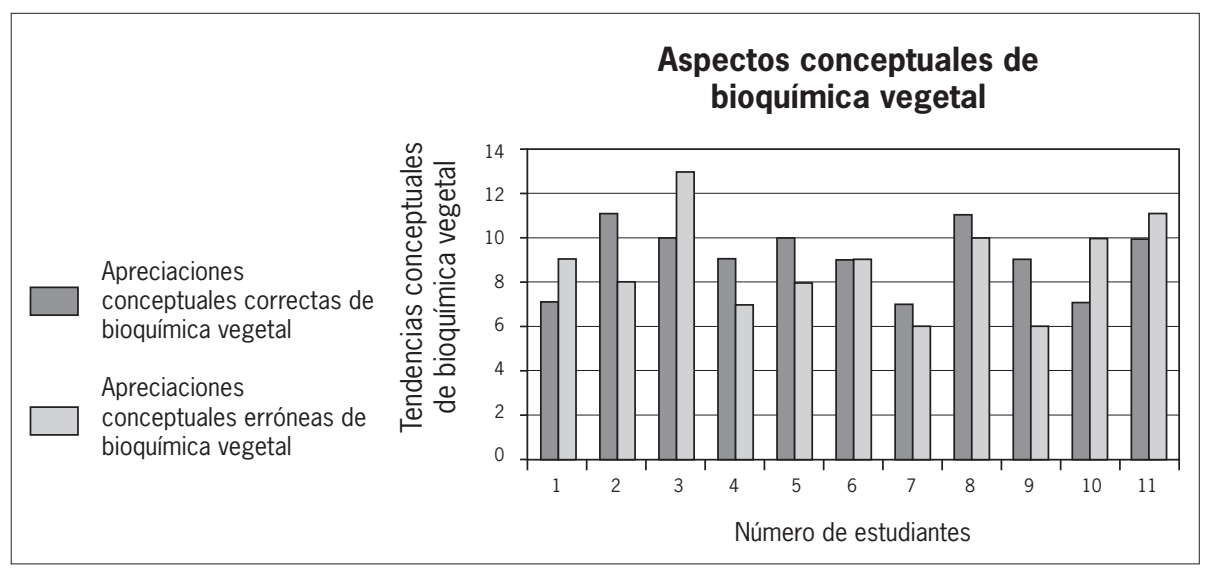

Figura 2. 
En la figura 3 se muestran las puntuaciones totales de las afirmaciones negativas y positivas de acuerdo con cada estudiante para la categoría de análisis 3 y según la tabla 1. Llama la atención que, a diferencia de los resultados obtenidos en la categoría 1 y 2 , la mayoría de los estudiantes (8) presenta valoraciones positivas en cuanto al conocimiento del glifosato y sus implicaciones ambientales (11-15), así mismo,
7 de ellos tienen valoraciones mayores o iguales a 11 en relación con las afirmaciones negativas, lo que confirma, sin ambigüedades, su conocimiento frente al problema ambiental que causa las fumigaciones con glifosato. Estos resultados son importantes, dado que favorecen el desarrollo de la estrategia, con respecto al problema ambiental seleccionado, ya que a los estudiantes les interesa el estudio del tema.

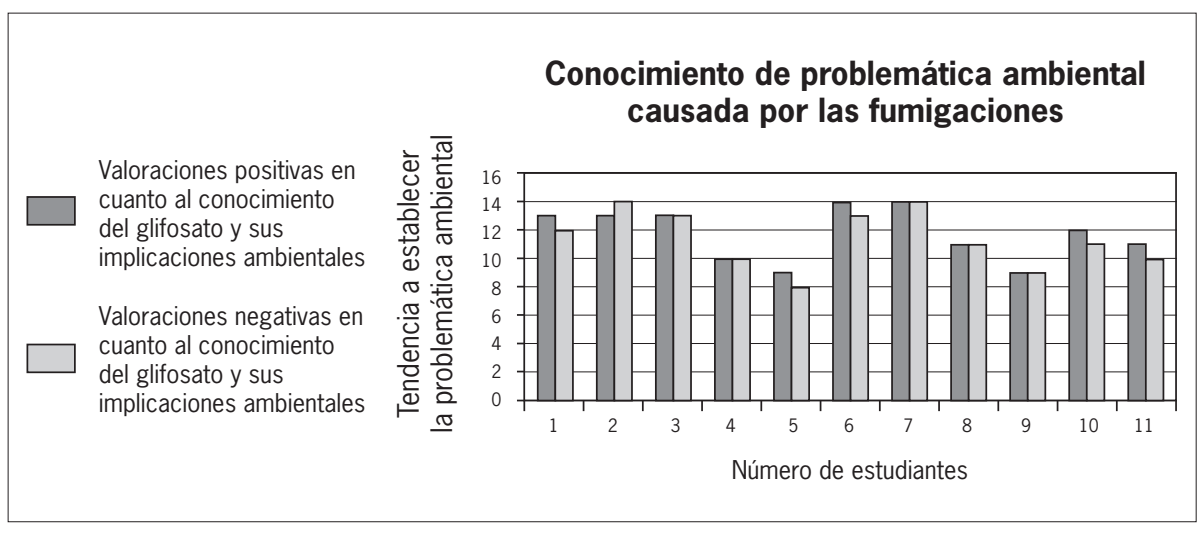

Figura 3.

En el desarrollo de una estrategia didáctica conviene rescatar los intereses de los estudiantes para el estudio de un tema determinado; en gran parte, éste es un punto inicial para que su componente afectivo y emocional favorezca un mejor aprendizaje. No obstante, la tendencia de los estudiantes a establecer apreciaciones inadecuadas de bioquímica vegetal sustenta la importancia que tiene la integración curricular del enfoque CTS para abordar los contenidos de bioquímica, y en consecuencia, con los siguientes puntos expuestos por Membiela (1997):
- El contenido debe ser aplicable a la vida.

- El desarrollo del tema contribuye al desarrollo cognitivo y a la madurez social del estudiante.

- El tema es importante en el mundo actual para los estudiantes, y probablemente permanecerá como tal para una proporción significativa de ellos durante su vida.

- Es preciso la aplicación de conocimientos en contextos distintos de los científicos escolares.

- Los estudiantes deben mostrar interés y entusiasmo hacia el tema. 


\section{Segunda etapa}

Teniendo presente los resultados obtenidos con la aplicación del instrumento 1, se procedió a desarrollar la estrategia con la construcción de los casos simulados, para lo cual se elaboró una guía (véase anexo 3) que orienta la controversia, y se preparó material magnético con información acerca de: la identificación del herbicida, los surfactantes, la ficha técnica del glifosato, la erradicación aérea de cultivos ilícitos de coca en Colombia, los efectos sobre salud y ambiente de los plaguicidas con glifosato, un reporte del impacto ambiental en las fronteras de Colombia y un estudio del metabolismo secundario del ácido shikímico.

Para el desarrollo de los casos simulados se organizaron los estudiantes en cuatro grupos:

- Grupo 1 Moderador (docentes y cinco estudiantes): comunidad académica (lectura de noticias reales y disertaciones conceptuales).

- Grupo 2 (cuatro estudiantes): representantes de la empresa productora del herbicida (algunos aspectos químicos del glifosato y su toxicología).

- Grupo 3 (cinco estudiantes): representantes del gobierno (políticas sobre fumigaciones de cultivos ilícitos).

- Grupo 4 (cuatro estudiantes): representantes de la salud, medio ambiente y afectados (impacto ambiental).

Tanto en el desarrollo de la controversia como en los informes entregados, cada grupo discutió y contrastó sus apreciaciones con los demás grupos, destacándose los siguientes aspectos por grupo, que fueron tomados tanto de las grabaciones del caso simulado, como de los informes presentados por cada actor:

Grupo 1. Este grupo lee las noticias presentadas en el anexo 2, y además socializa la guía que orienta el caso simulado. El papel principal de este grupo es moderar la discusión y propiciar el establecimiento de acuerdos entre los actores.

Grupo 2. Los estudiantes que pertenecen a este grupo explican el significado químico del glifosato y la manera como se comercializa el producto; para esto señalan que el glifosato es un herbicida no selectivo de acción sistémica, de amplio espectro, y adecuado para el control de muchas especies de malezas, en tratamientos de pos-emergencia al follaje. No actúa sobre las semillas que existan por debajo del suelo y tampoco es absorbido por las raíces. En igualdad de condiciones, también se puede decir que no es de acción residual prolongada y que no es ni actúa como herbicida esterilizante del suelo.

La casi totalidad de las formulaciones comerciales del glifosato son fáciles de manejar, muy solubles en agua y químicamente muy estables en cualquier proporción. A lo anterior se adiciona la baja tensión de vapor, lo cual significa que las formulaciones de uso en el campo no sean volátiles.

De acuerdo con sus propiedades fisicoquímicas, se puede decir que es un plaguicida perteneciente al grupo de los herbicidas de acción sistémica, por la vía del follaje. No es apto para tratamientos de control de malezas por la vía del sistema radicular. 
El glifosato es una solución líquida, clara, viscosa y de color ambarino; normalmente tiene un $\mathrm{pH}$ de 4,4 a 4,9 y una gravedad específica de 1,17. Prácticamente inoloro o con un ligero olor a amina; tiene un peso molecular de $169,08 \mathrm{~g} / \mathrm{mol}$ y un punto de fusión de $200^{\circ} \mathrm{C}$. El glifosato se comercializa en forma de concentrados solubles de la sal isopropanolamina del N- (Fosfonometil) glicina, donde se integran el glifosato y los ingredientes inertes requeridos para cada tipo de formulación comercial.

De otra parte, exponen los efectos sobre la salud y el ambiente, señalando que el manejo de este producto está expuesto en las indicaciones que hace la empresa, en relación con su toxicología, antídotos y procedimientos adecuados para su aspersión.

Grupo 3. Este grupo argumenta que han existido estudios de impacto ambiental que se han realizado en cooperación con agencias norteamericanas como la Enviromental Proteccion Agency y el Departamento de Estado de los Estados Unidos que señalan que la toxicidad para el ser humano y el ambiente no es altamente peligrosa. Además, sustentan las normas de seguridad y los equipos que se han utilizado en la fumigación en Colombia.

Grupo 4. Este grupo señala las denuncias que se han presentado por las fumigaciones, exponiendo la situación desde épocas anteriores; es así como el grupo indica que desde la década de los ochenta se comenzaron a realizar fumigaciones aéreas con glifosato. Esto ha provocado innumerables denuncias nacionales e internacionales debido a los impactos ambientales y en la salud humana. Según varias denuncias realizadas en 1992, se sustenta que las fumigaciones han provocado enfermedades respiratorias y cutáneas en varias personas. Así mismo, se han afectado cultivos de sustento de varias familias campesinas y han muerto animales domésticos. Igualmente, se muestra con estudios la destrucción de ecosistemas como es el caso de la Sierra Nevada de Santa Marta.

Coherente con lo anterior, para el año 2002 persistían las denuncias. Por ejemplo, en el Putumayo se registraron 6.553 quejas según registra un informe de la Defensoría del Pueblo. Además, varias ONG y científicos del mismo Estados Unidos han criticado firmemente la certificación del gobierno estadounidense.

Los argumentos de los gobiernos de Estados Unidos y Colombia para continuar con las fumigaciones son la lucha contra el narcotráfico. Según ellos, los cultivos ilícitos son los responsables de la pobreza y la violencia e incluso del deterioro ambiental; no obstante, las comunidades y los científicos han propuesto otras vías para la erradicación, como la manual, el control biológico, el control químico directo, con todas las precauciones necesarias y otras alternativas que puedan emerger de la investigación.

En Colombia continúan las fumigaciones, destacándose la aplicación de este herbicida por vía aérea en los departamentos del Putumayo y Norte de Santander. En el primero se sabe que el Plan Putumayo contempla inversiones de 300 mil millones de pesos. 
Después que los grupos realizan sus presentaciones, el grupo moderador profundiza la discusión teniendo en cuenta los siguientes aspectos:

- Explicación bioquímica de la actividad del glifosato en la plantas, sin importar si es amapola o marihuana; para tal fin, se discute la síntesis de aminoácidos aromáticos por medio de la ruta del ácido shikímico y su relación con otras rutas. El grupo explica los esquemas que se muestran en el anexo 4.

- Mayor estudio de los problemas mencionados en las noticias.

- Establecimiento de acuerdos y compromisos concretos de cada actor, que garanticen el respeto de los derechos de los ciudadanos y la conservación del ambiente.

Alrededor de estos aspectos los actores discuten y establecen algunos acuerdos generales:

- Es necesario que el Gobierno se comprometa a revisar la política nacional de fumigaciones, lo que implica rigurosos estudios de impacto ambiental y sanitario.

- La compañía debe adelantar programas de formación para los usuarios del producto que garanticen un adecuado manejo de los aspectos toxicológicos y ambientales del glifosato. Así mismo, apoyará estudios de impacto ambiental y sanitario.

- Las comunidades se comprometen a revisar los estudios realizados y a ejercer el papel de veeduría ciudadana en todo el proceso.

- La comunidad académica adelantará procesos de cualificación a los actores sociales, que redunden en comprender las implicaciones bioló- gicas, químicas, físicas y ambientales que implican las fumigaciones. De la misma forma, llevará a cabo investigaciones que permitan recomendar el uso o la suspensión de las fumigaciones.

La evaluación de la estrategia se realizó de manera cualitativa mediante el análisis de las grabaciones que se hicieron durante los casos simulados. Para la presentación de estos análisis se estableció una síntesis de los aspectos detectados en las tres categorías de los instrumentos usados en la primera etapa, apreciando los siguientes avances:

\section{Establecimiento de relaciones CTSA por parte de los estudiantes que representan a los actores (categoría 1)}

Los estudiantes, en la representación de cada actor, reconocen que el problema ambiental y sanitario planteado en las noticias requiere análisis de distintos puntos de vista; entre ellos es importante el aporte de la ciencia, dado que ésta arroja elementos para saber la composición, actividad, toxicología y precauciones acerca del uso del glifosato.

Así mismo, se destacan las relaciones entre ciencia, sociedad y ambiente, a partir del estudio del problema de las fumigaciones, teniendo presente que esta actividad social tiene importantes implicaciones en el ambiente y en la salud de los ciudadanos.

De otra parte, se valora que la participación de los actores en la controversia pública y las decisiones que puedan tomarse requieren una adecuada alfabetización científica, entendida como el conocimiento de aspectos básicos de la ciencia, la tecnología y el ambiente que permitan comprender un problema concreto 
que afecta la comunidad, como es el caso de las fumigaciones con glifosato.

\section{Apreciaciones conceptuales de bioquímica vegetal (categoría 2)}

El desarrollo de la controversia pública facilitó que los estudiantes comprendieran la actividad bioquímica del glifosato, ya que con solvencia explican la inhibición enzimática causada por el herbicida en la ruta metabólica del ácido shikímico, así mismo, establecen relaciones entre rutas metabólicas de las plantas, tales como la vía de las pentosas fosfato y el ciclo de Calvin. La explicación bioquímica les permitió comprender que el herbicida actúa sobre cualquier vegetal, sin discriminar si es un cultivo ilícito, lo cual devela implicaciones ambientales.

\section{Problemática ambiental causada por las fumigaciones (categoría 3)}

Las presentaciones en la controversia por parte de los estudiantes evidencian el notable interés de los participantes frente al estudio de problemas ambientales y sanitarios generados por las fumigaciones. Esto se manifiesta en las intervenciones que presentan el desarrollo histórico de algunas denuncias hechas por comunidades y grupos ambientales frente a las fumigaciones con glifosato.

\section{Conclusiones}

- El enfoque CTSA es una alternativa diferente y apropiada mediante la cual el proceso de enseñanza-aprendizaje de las ciencias ya no se concibe como un proceso memorístico, sino, más bien, como una estrategia motivadora y enriquecedora para el estudiante.

- Las unidades curriculares, pensadas desde un enfoque CTSA, generan actitudes de formación personal en relación con el ambiente y la calidad de vida; permiten al estudiante tomar decisiones con respecto a las temáticas desarrolladas, teniendo en cuenta aspectos científicos, técnicos, económicos y políticos; a su vez, promueven la participación individual y social de los estudiantes de una manera responsable y autónoma.

- El desarrollo de la estrategia didáctica, basada en la metodología de casos simulados, posibilita el desarrollo de controversias públicas de importante relevancia social en donde los estudiantes asumen roles de diferentes actores sociales que contribuyen en su formación como ciudadanos responsables en la toma de decisiones, al igual que favorece el aprendizaje de aspectos de bioquímica vegetal en relación con la actividad química del glifosato en las plantas.

- Las simulaciones CTSA son una alternativa educativa para propiciar el aprendizaje social y la participación en las controversias tecnocientíficas.

- Adquirir una visión adecuada de los problemas en que está inmersa la humanidad actualmente, sus causas y las medidas necesarias que se deben adoptar para darles solución, es un compromiso irrenunciable de la formación de profesores en ciencias y en particular, de química. $\boldsymbol{\Delta}$ 


\section{Anexos \\ Anexo 1. Instrumento 1
UNIVERSIDAD PEDAGÓGICA NACIONAL, FACULTAD DE CIENCIA Y TECNOLOGÍA DEPARTAMENTO DE QUIMICA - MAESTRÍA EN DOCENCIA DE LA QUÍMICA ${ }^{2}$ INSTRUMENTO 1

Edad: Sexo: Facultad Dpto:

Proyecto curricular Semestre Fecha:

Respetado estudiante: a continuación se presentan algunos ítems y preguntas que están relacionados con temáticas de la dinámica social del país y de ciertos aspectos de bioquímica. Conteste con libertad y de manera autónoma. Agradecemos su colaboración y atención.

1.Para cada una de las afirmaciones que relacionan aspectos fundamentales en el contexto Ciencia, Tecnología, Sociedad y Ambiente marque en las casillas numéricas una $\mathrm{X}$ según las siguientes opciones:

1. Completamente en desacuerdo. 3. No sé qué decir.

2. En desacuerdo.

\section{De acuerdo.}

5. Completamente de acuerdo.

\begin{tabular}{|c|c|c|c|c|c|}
\hline Afirmaciones & 1 & 2 & 3 & 4 & 5 \\
\hline \multicolumn{6}{|l|}{$\begin{array}{l}\text { 1. Se debe tomar conciencia sobre los resultados de las investigaciones, aplicaciones tecnoló- } \\
\text { gicas y las acciones que la industria desarrolla para solucionar las necesidades y demandas } \\
\text { de la sociedad sin perjudicar el ambiente. }\end{array}$} \\
\hline \multicolumn{6}{|l|}{$\begin{array}{l}\text { 2. El desarrollo científico y tecnológico ha implicado el progreso social, al igual que la altera- } \\
\text { ción ambiental. }\end{array}$} \\
\hline \multicolumn{6}{|l|}{$\begin{array}{l}\text { 3. El avance acelerado de los conocimientos científicos y tecnológicos está provocando un } \\
\text { estado de alerta y ciertos temores en la sociedad en general }\end{array}$} \\
\hline \multicolumn{6}{|l|}{$\begin{array}{l}\text { 4. La ciencia es una producción cultural, histórica y comunitaria que puede beneficiar o afectar } \\
\text { a la sociedad. }\end{array}$} \\
\hline \multicolumn{6}{|l|}{$\begin{array}{l}\text { 5. La ciencia es una empresa autónoma, objetiva y neutral que no se afecta por los problemas } \\
\text { sociales. }\end{array}$} \\
\hline \multicolumn{6}{|l|}{$\begin{array}{l}\text { 6. El progreso social depende del crecimiento económico, éste depende del desarrollo } \\
\text { tecnológico, y éste, a su vez, depende del desarrollo sin interferencias políticas o sociales del } \\
\text { conocimiento científico. }\end{array}$} \\
\hline \multicolumn{6}{|l|}{$\begin{array}{l}\text { 7. La ruta de aminoácidos aromáticos no está presente en las formas de vida de los mamíferos, } \\
\text { aves o fauna acuática, lo que explica la acción selectiva del glifosato en plantas y su baja } \\
\text { toxicidad en mamíferos. }\end{array}$} \\
\hline \multicolumn{6}{|l|}{$\begin{array}{l}\text { 8. La formación de metabolitos primarios, intermediarios y secundarios ocurre a través de } \\
\text { reacciones enzimáticas. }\end{array}$} \\
\hline \multicolumn{6}{|l|}{$\begin{array}{l}\text { 9. Tanto las plantas como los animales presentan rutas metabólicas que producen aminoáci- } \\
\text { dos aromáticos. }\end{array}$} \\
\hline \multicolumn{6}{|l|}{$\begin{array}{l}\text { 10. El ácido shikímico es el precursor de la mayoría de constituyentes vegetales que contienen } \\
\text { anillos aromáticos diferentes de los formados en la ruta del acetato-malonato. }\end{array}$} \\
\hline \multicolumn{6}{|l|}{ 11.En muchas reacciones bioquímicas no son necesarios los procesos enzimáticos. } \\
\hline \multicolumn{6}{|l|}{$\begin{array}{l}\text { 12. Las plantas sólo fabrican aminoácidos aromáticos por la ruta metabólica del acetato-ma- } \\
\text { lonato. }\end{array}$} \\
\hline \multicolumn{6}{|l|}{$\begin{array}{l}\text { 13. Algunas organizaciones han denunciado el impacto ambiental y social de las fumigaciones } \\
\text { con glifosato debido a la falta de estudios ambientales por parte del gobierno. }\end{array}$} \\
\hline \multicolumn{6}{|l|}{$\begin{array}{l}\text { 14. El glifosato es un herbicida no selectivo que puede afectar otras especies vegetales dife- } \\
\text { rentes a las presenten en los cultivos ilícitos. }\end{array}$} \\
\hline \multicolumn{6}{|l|}{$\begin{array}{l}\text { 15. La aspersión de glifosato se lleva a cabo en Colombia bajo parámetros muy rígidos estable- } \\
\text { cidos por el Auditor Ambiental del gobierno de Colombia del programa de fumigaciones. }\end{array}$} \\
\hline \multicolumn{6}{|l|}{$\begin{array}{l}\text { 16. El glifosato tiene una actividad sistémica, lo que significa que circula por el sistema vascular } \\
\text { de la planta afectando toda la planta, no sólo el follaje tratado. }\end{array}$} \\
\hline \multicolumn{6}{|l|}{ 17. El glifosato utilizado en las plantaciones forestales es inofensivo. } \\
\hline & & & & & \\
\hline
\end{tabular}

2 Instrumento diseñado por Leonardo F. Martínez y Álvaro P. Rojas Duarte. 
Anexo 2

\section{EL HERALDO \\ MAGDALENA}

BARRANOUILLA 22 DE JULIODE 1986

\section{Senado contra glifosato en Sierra Nevada}

Bogotá, jul. 21 (Colprensa). La Unión Patriótica en su primera proposición en el Senado presento hoy el proyecto de ley reglamentario del acto legislativo número 1 de 1986 , sobre la elección de alcaldes y consulta popular.

De igual forma fue aprobada por la plenaria una proposición del partido liberal que será enviada al Gobierno Nacional para prohibir la fumigación de la Sierra Nevada de Santa Marta, con glifosato, con miras a destruir los cultivos de marihuana.

Parlamentarios liberales como Carlos Holmes Trujillo, Raúl Orejuela Bueno y Ernesto Samper han dicho que su partido promoverá el primer gran debate en el Senado antes que termine la presente administración, sobre el uso indiscriminado del químico en los parques naturales de Colombia.

\section{El Defensor y el glifosato}

Tomado de El Tiempo febrero de 2003.

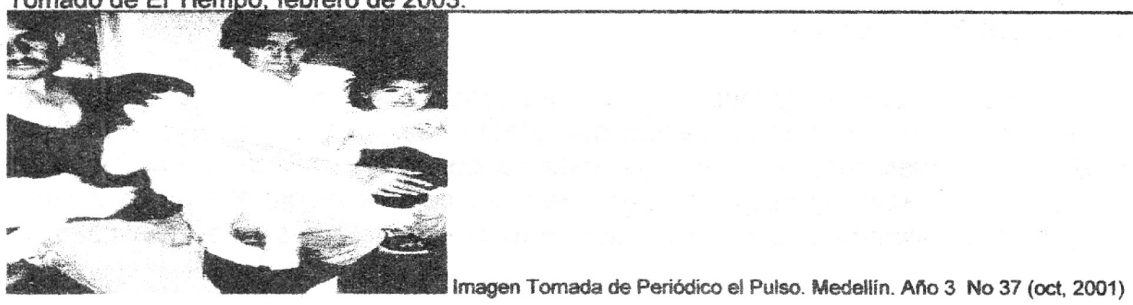

El defensor del Pueblo, Eduardo Cifuentes, dijo ayer que el aumento de la dosis de glifosato para la fumigación de cultivos ilícitos "se adelanta en condiciones de absoluta ilegalidad", pues ni siquiera se consolidó el estudio de los efectos sobre el medio ambiente y la salud humana de la dosis anterior, y la administración Uribe autorizó el incremento de ese tope. "Esta es una clara violación" aseguró.

\section{Indígenas pierden cultivos}

Tomado de El Tiempo, Miércoles 16 de abril de 2003

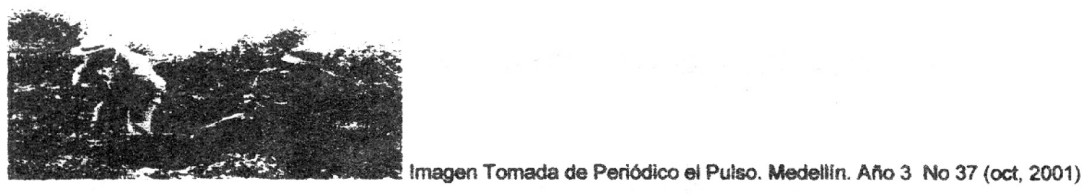

POPAYÁN. Cerca de 2.000 indigenas yanaconas del resguardo Río Blanco, en Sotará (Cauca), lanzaron ayer un SOS a los gobiernos regional y nacional por la pérdida total de cultivos de pan coger y cría de especies menores, como consecuencia de las aspersiones con glifosato en cultivos ilicitos de amapola. Según la Umata, las más afectadas son las veredas Pueblo Quemado, Salinas, La Floresta, Mambilona, Chapiloma y las Cabras. Los aborigenes dicen que no tienen cómo alimentar a sus familias. 


\section{Anexo 3 \\ UNIVERSIDAD PEDAGÓGICA NACIONAL, FACULTAD DE CIENCIA Y TECNOLOGÍA DEPARTAMENTO DE QUIMICA - MAESTRÍA EN DOCENCIA DE LA QUIMICA}

\section{GUÍA DE TRABAJO 3}

\section{ACTIVIDAD LA ERRADICACIÓN AÉREA DE CULTIVOS ILÍCITOS DE COCA EN COLOMBIA ${ }^{4}$}

\section{Objetivos}

- El aprendizaje de la participación en controversias sociales en el contexto de problemas derivados del desarrollo tecnocientífico

- Llevar al aula de clase los problemas de la flexibilidad interpretativa presentes en la ciencia

- Participar de forma efectiva en controversias reales del país, a partir de conocimientos sociales, científicos y ambientales.

\section{Metodología}

Los participantes forman cuatro grupos que representan las partes implicadas en el caso.

Grupo 1: Grupo moderador Comunidad académica (lectura de las noticias y disertaciones conceptuales). Grupo 2:Representantes de la empresa productora del herbicida (algunos aspectos químicos del glifosato y su toxicología)

Grupo 3:Representantes del Gobierno (Políticas sobre fumigaciones de cultivos ilícitos)

Grupo 4: Representantes de la salud, medio ambiente y afectados (impacto ambiental)

Tras la lectura conjunta de la noticia y de documentos complementarios con los que se articula el caso, se da inicio al caso simulado a través de los papeles de los actores sociales. Debe existir un actor social que hace de mediador y moderador encargado de presentar los distintos puntos de vista y de velar por la transparencia democrática del debate (grupo de estudiantes y profesores). Este grupo tiene la tarea de recomendar o no el uso del glifosato o proponer otras soluciones. Posteriormente el grupo moderador da comienzo a la intervención de los otros grupos que, a través de una exposición de 15 minutos y 5 minutos de preguntas aclaratorias, defiende su posición.

Finalmente el grupo moderador tendrá que determinar qué solución tomar, según las versiones presentadas. Cada grupo contará con actores que intervienen en el problema con perfiles definidos (científicos, ingenieros, empresas, asociaciones ecologistas, grupos vecinales, grupos políticos, asociaciones profesionales y ciudadanos afectados).

\section{Material de trabajo}

- Cuestionario Inicial y final para conocer las informaciones y las ideas alternativas de los estudiantes sobre el objeto de trabajo y contrastar posibles cambios.

- Archivos en formato PDF:Identificación del herbicida, surfactantes, ficha técnica del glifosato. Grupo 2.

- Archivos en formato PDF:"La erradicación aérea de cultivos ilícitos de coca en Colombia". Grupo 3.

- Archivos Word: Efectos sobre salud y ambiente de plaguicidas con glifosato, ficha técnica del glifosato, reporte de impacto en las fronteras. Grupo 4.

Teniendo en cuenta que como futuros profesores de química debemos manejar los aspectos químicos que implican controversias públicas de interés social, es necesario que todos los grupos conozcan qué ocurre químicamente con el glifosato en las plantas, para esto se requiere estudiar las propiedades fisicoquímicas del glifosato y su correspondiente metabolismo. Con el propósito de apoyar tu estudio se anexan en archivo Word los siguientes documentos: Metabolismo secundario del ácido shikimico-Gr (esta es la principal ruta metabólica que afecta el glifosato dado que esta sustancia inhibe la enzima 5-enolpiruvilshikimato3fosfato sintetasa alterando la ruta metabólica del ácido shikimico que, a su vez, es un importante metabolito para la síntesis de aminoácidos de las plantas, lo cual es fatal para la planta terminando en la destrucción de su follaje y posteriorme en su muerte); caracterización fisicoquímica del glifosato y ficha técnica del herbicida. El Grupo 1, como es el moderador, debe conocer todas las temáticas.

\section{Evaluación}

Cada grupo debe entregar un informe de investigación elaborado por el grupo de trabajo, respecto al actor que están representando.

\section{Nota}

Todos los grupos deben estudiar las siguientes temáticas para desarrollar una discusión rigurosa:

a) Ruta metabólica del fosfato pentosa

b) Ciclo de calvin

c) Síntesis de aminoácidos aromáticos.

3 Guía diseñada por Leonardo F. Martínez y Álvaro P. Rojas Duarte.

4 Caso simulado de CTS (los casos simulados CTS consisten en la articulación educativa de controversias públicas relacionadas con desarrollos tecnocientíficos con implicaciones sociales o medioambientales). 


\section{Anexo 4}

Esquemas usados por los estudiantes para explicar las rutas metabólicas de las plantas y su alteración por el glifo- sato, específicamente en la biosíntesis de aminoácidos aromáticos.

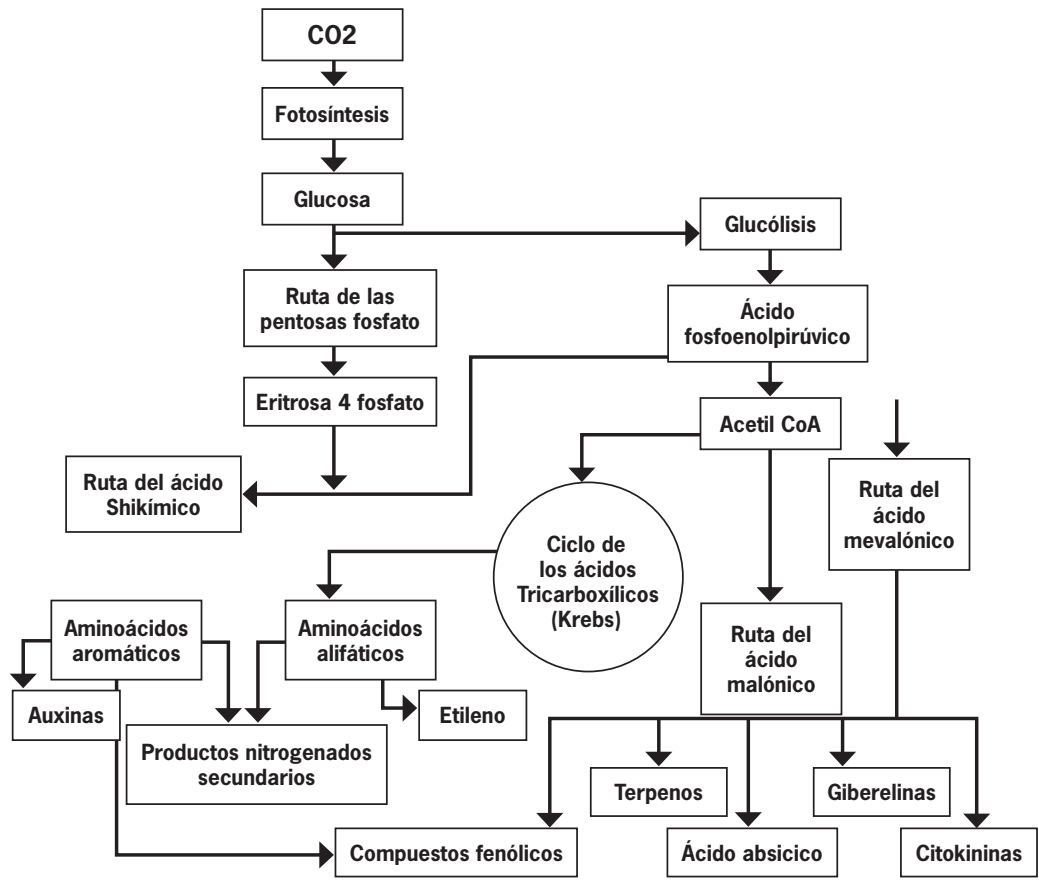

Vía del ácido shikímico

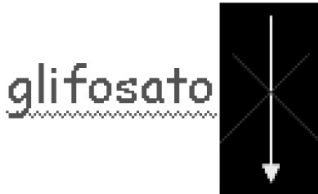

5-enolpiruvilshikimato-3-fosfato sintetasa

Síntesis de aminoácidos aromáticos
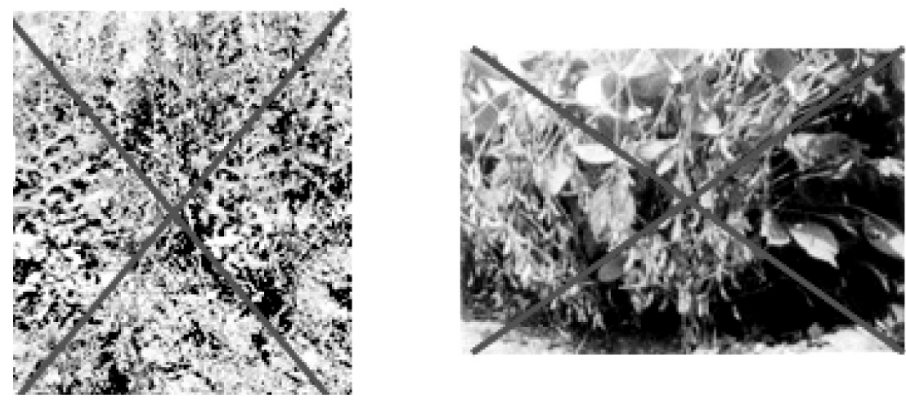


\section{Bibliografía}

Acevedo, J. (1996a). “Cambiando la práctica docente en la enseñanza de las ciencias a través de CTS”. Revista Borrador, 13:26-30. En línea en Sala de Lecturas CTS+I de la OEI, <http:// www.campus-oei.org/salactsi/acevedo2.htm>, 2001.

Blanco, A. y Uraga, C. (2001). "Materiales didácticos para un enfoque CTS: las bebidas”. Enseñanza de las Ciencias, número extra VI Congreso, pp. 49-50.

Bucat, R. (2005). "Implications of chemistry education research for teaching practice: pedagogical content knowledge as a way forward". Chemical Education International, Vol. 6 (1), 1-2. [Online] www.iupac.org/publications/cei

Campanario, J. (1999). “Cómo enseñar Ciencias: principales tendencias y propuestas". Enseñanza de las Ciencias, Vol. 17 (2), 179-192.

Carrascosa, J. (2005). "El problema de las concepciones alternativas en la actualidad (Parte I), análisis sobre las causas que la originan y/o mantienen”. Revista Eureka sobre enseñanza y divulgación de las ciencias, Vol. 2 (2), 183-208.

Catebiel, V. (2003). "Enseñanza de la química con un enfoque CTS: su vinculación con el cambio actitudinal de los estudiantes". En: revista de la Facultad de Ciencia y Tecnología Tecné, Episteme y Didaxis, número extra, pp. 181-183.

Del Rincón, Arnal, Latorre y Sans (1995). Técnicas de investigación en Ciencias Sociales. Madrid: Dykinson.

Edwards, M, Gil, D, Vilches, A. y Praia J. (2004). "La atención a la situación del mundo en la educación científica”.
Enseñanza de las Ciencias, Vol. 22 (1), 47-64.

Galavoskky, L y otros (2003). "Representaciones mentales, lenguajes y códigos en la enseñanza de las ciencias naturales. Un ejemplo para el aprendizaje del concepto de reacción química, a partir del concepto de mezcla”. Enseñanza de las Ciencias, Vol. 21 (1), 107-121.

Gallego, R. y Pérez, R. (1999). El problema del cambio en las concepciones epistemológicas, pedagógicas y didácticas. Santafé de Bogotá: Universidad Pedagógica Nacional.

Garritz, A. (1994). “Aportaciones y opiniones sobre la enseñanza de la química en el nivel medio y superior. CienciaTecnología-Sociedad: a diez años de iniciada la corriente movimiento". En línea en Sala de Lecturas CTS+I de la OEI, http://www.campus-oei.org

González, M. López, J. y Luján, J. (1997). Ciencia, tecnología y sociedad: lecturas seleccionadas. Barcelona: Ariel., pp. 5-13.

Gordillo, (2001). AIDS-2001: La vacuna contra el sida. Simulación educativa de un caso CTS sobre la salud. OEI.

. y Osorio, C. (2003). "Educar para participar en ciencia y tecnología un proyecto para la difusión de la cultura científica". Revista Iberoamericana de educación, (32), 165-210. . y Acevedo, J. (2005). “Acercando la ciencia a la sociedad: la perspectiva CTS su implementación educativa". En línea en Sala de Lecturas CTS +1 de la OEI, http://www.campusoei. org/salactsi/mmartin.htm

Guerrero, J. y Cabrera, L. (2004). "Estrategia didáctica para la enseñanza de la química en el contexto de relaciones 
ciencia, tecnología, sociedad y ambiente". En: Revista de la Facultad de Ciencia y Tecnología, Tecné, Epsíteme y Didaxis, (16), 132-145.

Guruceaga, A. y González, F. (2004). "Aprendizaje significativo y educación ambiental: análisis de los resultados de una práctica fundamentada teóricamente". Enseñanza de las Ciencias, Vol. 22, (1), 115-136.

Gutiérrez, R. (2004). "La modelización y los procesos de enseñanza/aprendizaje”. Alambique. (42), 8-18.

Jhonstone, A. (1997). "Chemistry Teaching-Science or Alchemy?" Journal of Chemical Education, Vol. 74(3), 262-268.

Libres, M. Comesaña, M. y Tojo, J. (2001). "La historia de la química en los libros de texto: una escasez escandalosa". Enseñanza de las Ciencias, número extra. VI Congreso, pp. 243-244.

Manassero, V. y Acevedo, J. (2001). "Avaluació dels temes de ciència, tecnologia i societat". Palma de Mallorca (España): Conselleria d'Educació i Cultura del Govern de les Illes Ballears. En línea con el título: "El movimiento, ciencia-tecnología-sociedad y la Enseñanza de las Ciencias", Sala de Lecturas CTS+I de la OEI, <http:// www.campus-oei.org/salactsi/acevedo13.htm>
Membiela, P. (1995). “Ciencia-TecnologíaSociedad en la enseñanza-aprendizaje de las Ciencias Experimentales". Alambique, (3), 7-11.

Membiela, I. (1997). "Una revisión del movimiento educativo ciencia-tecnología-sociedad. Enseñanza de las Ciencias, Vol. 15 (1), 51-57.

Moreira, M., Greca, I. y Rodríguez, L. (2004). "Modelos mentales y modelos conceptuales en la enseñanza-aprendizaje de las ciencias". En: Sobre cambio conceptual, obstáculos representacionales, modelos mentales, esquemas de asimilación y campos conceptuales. Porto Alegre: IF-UFRGS, pp. 44-66.

Solbes, J. y Vilches, A. (1992). "El modelo constructivista y las relaciones Ciencia/ Técnica/Sociedad (C/T/S)". Enseñanza de las Ciencias, Vol. 10 (2), 181-186. . (2004). "Papel de las relaciones entre ciencia, tecnología, sociedad y ambiente en la formación ciudadana". Enseñanza de las Ciencias, Vol. 22 (3), 337 -348.

Valderrama, A. y Jiménez, J. (2004). "Cómo abordar el tema de ciencia, tecnología y sociedad en la educación básica". En: Aula Urbana n ${ }^{\circ} 46$ (enero marzo) pp.12-14. 